hep-ph/0105061

BNL-HET-01/14, CERN-TH/2001-116

$\mathrm{DCPT} / 01 / 38, \mathrm{IPPP} / 01 / 19$

UMN-TH-2004/01, TPI-MINN-01/18

\title{
Observability of the Lightest CMSSM Higgs Boson at Hadron Colliders
}

\author{
John Ellis ${ }^{1}$, Sven Heinemeyer ${ }^{2}$, Keith A. Olive ${ }^{1,3}$ and Georg Weiglein ${ }^{1,4}$ \\ ${ }^{1}$ TH Division, CERN, Geneva, Switzerland \\ ${ }^{2}$ High-Energy Theory Group, Brookhaven National Laboratory, Upton, NY 11973, USA \\ ${ }^{3}$ Theoretical Physics Institute, School of Physics and Astronomy, \\ University of Minnesota, Minneapolis, MN 55455, USA \\ ${ }^{4}$ Institute for Particle Physics Phenomenology, University of Durham, \\ Durham DH1 3LR, UK
}

\begin{abstract}
We discuss the observability of the lightest neutral Higgs boson in the constrained MSSM (CMSSM), with universal soft supersymmetry-breaking parameters, at hadron colliders such as the Tevatron and the LHC. We take account of the constraints on parameter space provided by LEP, the measured rate of $b \rightarrow s \gamma$ decay, the cosmological relic density $\Omega_{\chi} h^{2}$, and the recent measurement of $g_{\mu}-2$. We normalize products of the expected CMSSM Higgs production cross sections and decay branching ratios $\sigma \times \mathcal{B}$ relative to those expected for a Standard Model Higgs boson of the same mass. In the $h \rightarrow \gamma \gamma$ channel, we find that $[\sigma(g g \rightarrow$ $h) \times \mathcal{B}(h \rightarrow \gamma \gamma)]_{\mathrm{CMSSM}} \gtrsim 0.85 \times[\sigma(g g \rightarrow h) \times \mathcal{B}(h \rightarrow \gamma \gamma)]_{\mathrm{SM}}$. In the $W^{ \pm} / \bar{t} t+h, h \rightarrow \bar{b} b$ channels, we find that $\left[\sigma\left(W^{ \pm} / \bar{t} t+h\right) \times \mathcal{B}(h \rightarrow \bar{b} b)\right]_{\mathrm{CMSSM}} \sim 1.05 \times\left[\sigma\left(W^{ \pm} / \bar{t} t+h\right) \times \mathcal{B}(h \rightarrow \bar{b} b)\right]_{\mathrm{SM}}$. We conclude that the lightest CMSSM Higgs boson should be almost as easy to see as the Standard Model Higgs boson: in particular, it should be discoverable with about $15 \mathrm{fb}^{-1}$ of luminosity at the Tevatron or $10 \mathrm{fb}^{-1}$ of luminosity at the LHC.
\end{abstract}

CERN-TH/2001-116

May 2001 
After the completion of the LEP experimental programme, which established a 95\% C.L. exclusion limit for the Standard Model (SM) Higgs boson of $m_{\mathrm{H}}>113.5 \mathrm{GeV}$ and gave a hint of a possible signal of a Higgs boson weighing $115 \mathrm{GeV}$ [1], the search for the Higgs boson shifts to hadron colliders, first the Tevatron and subsequently the LHC. There have been many studies of Higgs production and detection, both in the SM and in its minimal supersymmetric extension (MSSM). It just so happens that the existence of a Higgs boson with $m_{\mathrm{H}}=115 \mathrm{GeV}$ would offer the best prospects for the Tevatron collider and be the most challenging for the LHC. In the SM, the conclusions have been that, if the Higgs boson $H$ weighs $115 \mathrm{GeV}$, it might be discoverable at the 5 - $\sigma$ level with $15 \mathrm{fb}^{-1}$ of luminosity at the Tevatron collider [2] (which might be accumulated by 2007), whilst $10 \mathrm{fb}^{-1}$ at the LHC (corresponding to about one year of operation) should be enough to discover the Higgs boson, whatever its mass up to about $1 \mathrm{TeV}$ [3]. In the MSSM, one expects the lightest neutral Higgs boson $h$ to weigh $\lesssim 130 \mathrm{GeV}$ 《], but the detectability of MSSM Higgs bosons depends on other model parameters in addition to their masses. A complete survey of MSSM parameter space would be a very lengthy task, and attention has often focussed on particular squark mixing scenarios [5]. Again, the conclusions have been encouraging for the LHC regarding the detection of at least one Higgs boson, and there are also hopes of finding the lightest MSSM Higgs boson $h$ at the Tevatron collider [6].

In this paper, we discuss Higgs observability at the Tevatron and the LHC within the constrained MSSM (CMSSM), in which the soft supersymmetry-breaking parameters are assumed to be universal at some high GUT input scale H. In this case, the amount of squark mixing typically does not coincide with that often assumed in previous analyses of MSSM Higgs detectability at the LHC or the Tevatron [5, 6], and the underlying structure of the CMSSM gives rise to a correlation between the parameters $m_{\mathrm{A}}$, the mass of the $\mathcal{C P}$ odd Higgs boson, and $\tan \beta$, the ratio of the vacuum expectation values of the two Higgs doublets, which in lowest order determine the Higgs boson phenomenology. As a new element in the discussion of the observability of the lightest $\mathcal{C P}$-even Higgs boson, we introduce the most up-to-date set of experimental and cosmological constraints on the CMSSM parameter space, including those from LEP [1], $b \rightarrow s \gamma$ [7, 8], cosmological dark matter [9, 10] and the anomalous magnetic moment of the muon [11, 12]. Whilst in the unconstrained MSSM the detectability of the lightest $\mathcal{C P}$-even Higgs boson is not guaranteed at the LHC even with $300 \mathrm{fb}^{-1}$ [5], both the CMSSM universality assumption and the restrictions on the CMSSM

\footnotetext{
${ }^{1}$ An economical way to ensure this universality is by gravity-mediated supersymmetry breaking in a minimal supergravity (mSUGRA) scenario, but there are other ways to validate the CMSSM assumptions, including no-scale supergravity scenarios.
} 
parameter space imposed by the above constraints reduce substantially the uncertainty in the detectability of MSSM Higgs bosons at hadron colliders, as we shall see.

The principal mechanisms for light Higgs boson production at hadron colliders considered in this paper are $g g \rightarrow$ Higgs [13] followed by Higgs $\rightarrow \gamma \gamma$ [14] and associated $\bar{t} t+$ Higgs production followed by Higgs $\rightarrow \bar{b} b$, which are of interest at the LHC [3], and $W^{ \pm *} \rightarrow$ $W^{ \pm}+$Higgs 15 followed by Higgs $\rightarrow \bar{b} b$, which is of interest at the Fermilab Tevatron collider [2].

A priori, the $\gamma \gamma$ signal of interest to the LHC is the most model-dependent, since it involves loop diagrams in both the $g g$-Higgs production vertex and the Higgs- $\gamma \gamma$ decay vertex. Fermion and boson loops contribute with opposite signs [14, raising the spectre of cancellations, e.g., for particular values of the stop masses and mixing parameters. The signal also depends inversely on the rate for Higgs $\rightarrow \bar{b} b$, which is the dominant decay mode in the mass range of interest. This can be enhanced in the MSSM, particularly for large $\tan \beta$, offering the danger of a further suppression in $\mathcal{B}(h \rightarrow \gamma \gamma)$. On the other hand, the Higgs- $\bar{t} t$ vertex is relatively model-independent, since the region of very small $\tan \beta$ is experimentally disfavoured. Moreover, the MSSM enhancement of the $h \bar{b} b$ vertex actually improves the branching ratio for $h \rightarrow \bar{b} b$, so the $\bar{t} t+h, h \rightarrow \bar{b} b$ signal at the LHC should be relatively secure.

In the case of the $W^{ \pm *} \rightarrow W^{ \pm}+h, h \rightarrow \bar{b} b$ signature of interest at the Fermilab Tevatron collider [2], it is known that the $W^{ \pm} W^{\mp} h$ vertex is generically suppressed in the MSSM relative to the $\mathrm{SM}$ by a factor $\sin ^{2}(\beta-\alpha)$. However, as we discuss in more detail later, this suppression does not occur in the CMSSM, at least in the preferred parameter range that is compatible with all the experimental and cosmological constraints. This observation, combined with the MSSM enhancement of the $h \bar{b} b$ vertex, suggests a priori that the prospects for $h$ detection via this signature should be no worse than in the SM.

We find in this paper that, in the allowed domain of CMSSM parameter space, $[\sigma(g g \rightarrow$ $h) \times \mathcal{B}(h \rightarrow \gamma \gamma)]_{\mathrm{CMSSM}} \gtrsim 0.85 \times[\sigma(g g \rightarrow h) \times \mathcal{B}(h \rightarrow \gamma \gamma)]_{\mathrm{SM}}$. Values as low as 0.5 would be allowed if one relaxed the $g_{\mu}-2$ constraint, in which case $\mu<0$ would be permitted, and furthermore abandoned the $b \rightarrow s \gamma$ constraint, for example when $\tan \beta=35, A_{0}=+m_{1 / 2}$ and $\mu<0$. In the $W^{ \pm}+h, h \rightarrow \bar{b} b$ and $\bar{t} t+h, h \rightarrow \bar{b} b$ channels, we find the expected result that $\left[\sigma\left(W^{ \pm} / \bar{t} t+h\right) \times \mathcal{B}(h \rightarrow \bar{b} b)\right]_{\mathrm{CMSSM}} \sim 1.05 \times\left[\sigma\left(W^{ \pm} / \bar{t} t+h\right) \times \mathcal{B}(h \rightarrow \bar{b} b)\right]_{\mathrm{SM}}$, because of the enhancement in the $\mathcal{B}(h \rightarrow \bar{b} b)$ over its value in the SM.

Before describing these results in detail, we first review our treatment of the experimental and cosmological constraints on the CMSSM parameter space.

There are interesting constraints from LEP on sleptons, charginos and stops, but the 
most relevant is that on the Higgs boson itself. In fact, within the CMSSM, the $b \rightarrow s \gamma$ and $g_{\mu}-2$ constraints overshadow the slepton constraint, so we do not discuss it further. The chargino constraint is also overshadowed, except (among the cases we study) for the choice $\tan \beta=10, \mu>0$. The LEP (and Tevatron collider) constraints on stops are also important in the general MSSM context, but not in the CMSSM discussed here. The LEP Higgs constraint within the $\mathrm{SM}$ is that $m_{\mathrm{H}}>113.5 \mathrm{GeV}$, and, as is well known, there is a hint of a signal with mass $115.0_{-0.9}^{+1.3} \mathrm{GeV}$ [四]. In contrast to the unconstrained MSSM, for which the $Z^{0} Z^{0} h$ coupling is strongly suppressed by $\sin ^{2}(\beta-\alpha)$ in a significant part of the parameter space, this coupling is very close to that of the SM Higgs for almost all possible parameter values in the CMSSM. We find a sizeable suppression of this coupling only for $\mu<0$, an option disfavoured by the $g_{\mu}-2$ constraint [12], in small parameter regions with large $\tan \beta$ and small $m_{1 / 2}$ and $m_{0}$. As a consequence, the SM limit ('observed' value) can be carried over to the CMSSM for most of the parameter space. We allow only CMSSM parameter choices that are consistent with $m_{\mathrm{h}}>113 \mathrm{GeV}$ in this case, so as to make some allowance for theoretical uncertainties in the calculation of $m_{\mathrm{h}}$ in the CMSSM. We give special consideration to the range $m_{\mathrm{h}} \sim 115 \mathrm{GeV}$, but do not impose any experimental upper limit on the CMSSM Higgs mass. For the regions with a significant suppression of the $Z^{0} Z^{0} h$ coupling, we apply the bound $m_{\mathrm{h}}>91.0 \mathrm{GeV}$.

The theoretical uncertainties in the CMSSM Higgs mass calculations are at present dominated by the experimental error in the mass of the top quark, since $\delta m_{\mathrm{h}} / \delta m_{\mathrm{t}}=\mathcal{O}(1)$. In our analysis below we use as default $m_{\mathrm{t}}=175 \mathrm{GeV}$, but also study the consequences if $m_{\mathrm{t}}=170$ or $180 \mathrm{GeV}$.

In the treatment of $b \rightarrow s \gamma$, we follow [10] in our implementation of NLO QCD corrections at large $\tan \beta[8]$. We assume the $95 \%$ confidence-level range $2.33 \times 10^{-4}<\mathcal{B}(b \rightarrow s \gamma)<$ $4.15 \times 10^{-4}$ [7], and we accept all CMSSM parameters sets that give predictions in this range, allowing for the scale and model dependences of the QCD calculations.

We assume $R$ parity conservation, so that the lightest supersymmetric particle (LSP) is stable. The LSP is expected in the CMSSM to be the lightest neutralino $\chi$, and may have an interesting cosmological relic density $\Omega_{\chi} h^{2}$. The regions of the CMSSM parameter space allowed by cosmology are taken from [10], where up-to-date results for large $\tan \beta$ are presented. We accept CMSSM parameter sets that have $0.1 \leq \Omega_{\chi} h^{2} \leq 0.3$. Lower values of $\Omega_{\chi} h^{2}$ would be allowed if not all the cosmological dark matter is composed of neutralinos. However, larger values of $\Omega_{\chi} h^{2}$ are excluded by cosmology.

The final constraint that we implement is that on the possible supersymmetric contribution $\delta a_{\mu}$ to the muon anomalous magnetic dipole moment $g_{\mu}-2 \equiv 2 a_{\mu}$, which we calculate 
as in [12]. The signal for non-zero $\delta a_{\mu}=(43 \pm 16) \times 10^{-10}$ is considered to be a 2.6- $\sigma$ effect [11], and we consider as preferred the $2-\sigma$ range $11 \times 10^{-10}<\delta a_{\mu}<75 \times 10^{-10}$. More conservatively, one might simply require $\delta a_{\mu} \geq 0$, which is sufficient largely to exclude the $\mu<0$ scenarios we discuss below, that are the only ones for which we find a substantial suppression of $[\sigma(g g \rightarrow h) \times \mathcal{B}(h \rightarrow \gamma \gamma)]_{\mathrm{CMSSM}}$.

For the evaluation of the cross sections and branching ratios, we use the programs FeynHiggs [16], which contains the diagrammatic results for the complete one-loop and dominant two-loop corrections to the Higgs-boson propagators [17], and HDECAY [18]. The supersymmetric parameters at the weak scale have been determined from the parameters at the GUT scale using the two-loop renormalization-group equations of [19]. Since FeynHiggs uses internally the physical (i.e. pole) masses of the squarks, it was necessary to convert to them from the $\overline{\mathrm{DR}}$ parameters (see also [20]). This was done by running all parameters in the mass matrices of the scalar top and bottom quarks down to a renormalization scale equal to the largest of the soft-breaking parameters in each mass matrix (when evaluated at their own scales). The mixing matrices were then diagonalized at this scale to yield the squark masses and mixing angles, which were then transformed into the corresponding on-shell parameters.

We present our results in $\left(m_{1 / 2}, m_{0}\right)$ planes for the choices $\tan \beta=10,50$ for $\mu>0$, consistent with $g_{\mu}-2$, and $\tan \beta=10,35$ for $\mu<0$. We do not consider values of $\tan \beta$ below 10 , since in the CMSSM the low-tan $\beta$ region is severely constrained by the experimental bound on the Higgs-boson mass f.

We first consider the signal for $\sigma(g g \rightarrow h) \times \mathcal{B}(h \rightarrow \gamma \gamma)$, starting with the default case $A_{0}=0$ and $m_{\mathrm{t}}=175 \mathrm{GeV}$ shown in Fig. 1. Panels (a) and (b) are for $\mu>0$ and $\tan \beta=10$ and 50, respectively, where the $\pm 2-\sigma$ limits from $g_{\mu}-2$ are shown as diagonal (red) solid lines. Panels (c) and (d) are for $\mu<0$ and $\tan \beta=10$ and 35, respectively, and there are no $g_{\mu}-2$ contours because this sign of $\mu$ is inconsistent with the measured value of $g_{\mu}-2$. The (near-)vertical (black) solid, dotted and dashed lines in all the panels of Fig. 1 correspond to $m_{\mathrm{h}}=113,115,117 \mathrm{GeV}$, that we take as the absolute lower limit, an indicative value and an indicative upper limit on $m_{\mathrm{h}}$, respectively ${ }^{\text {? }}$. The (pink) shaded regions are excluded by $b \rightarrow s \gamma$, and the (brown) bricked regions are excluded because the LSP is the lighter $\tilde{\tau}$ slepton. The cosmological region where $0.1 \leq \Omega_{\chi} h^{2} \leq 0.3$ is divided into different ranges of $\sigma(g g \rightarrow h) \times \mathcal{B}(h \rightarrow \gamma \gamma)$, relative to the SM value, that are (coloured) shaded differently as

\footnotetext{
${ }^{2}$ This is in contrast to the unconstrained MSSM, where for $m_{\mathrm{t}}=174.3 \mathrm{GeV}$ values as low as $\tan \beta=3$ are allowed [21].

${ }^{3}$ Compared to earlier analyses, such as $[10]$, the $m_{h}$ contours appear at lower $m_{1 / 2}$ values. This is due to an improved Higgs mass calculation [16, 17], where an upward shift in $m_{h}$ for given $\left(m_{1 / 2}, m_{0}\right)$ of $\sim 1.5$ to $\sim 4 \mathrm{GeV}$ is possible.
} 
indicated in each panel.

We see in panel (a) of Fig. 1 1 that $0.85 \leq[\sigma(g g \rightarrow h) \times \mathcal{B}(h \rightarrow \gamma \gamma)]_{\mathrm{CMSSM}} /[\sigma(g g \rightarrow$ $h) \times \mathcal{B}(h \rightarrow \gamma \gamma)]_{\mathrm{SM}} \leq 1.00$ for $\mu>0$ and $\tan \beta=10$, once one imposes $m_{\mathrm{h}} \geq 113 \mathrm{GeV}$ and $0.1 \leq \Omega_{\chi} h^{2} \leq 0.3$. These same constraints impose $0.90 \leq[\sigma(g g \rightarrow h) \times \mathcal{B}(h \rightarrow$ $\gamma \gamma)]_{\mathrm{CMSSM}} /[\sigma(g g \rightarrow h) \times \mathcal{B}(h \rightarrow \gamma \gamma)]_{\mathrm{SM}} \leq 1.00$ for $\mu>0$ and $\tan \beta=50$, as seen in panel (b). Here the lower limit on $m_{1 / 2}$ from $b \rightarrow s \gamma$ is stronger than that from $m_{\mathrm{h}}$, but does not change the lower bound on the $h \rightarrow \gamma \gamma$ signal. Note that the BNL $g_{\mu}-2$ constraint imposes $[\sigma(g g \rightarrow h) \times \mathcal{B}(h \rightarrow \gamma \gamma)]_{\mathrm{CMSSM}} /[\sigma(g g \rightarrow h) \times \mathcal{B}(h \rightarrow \gamma \gamma)]_{\mathrm{SM}} \leq 0.93(0.96)$ for $\tan \beta=10(50)$.

In panel (c), for $\mu<0$ and $\tan \beta=10$, the $m_{\mathrm{h}}$ constraint again imposes $0.85 \leq[\sigma(g g \rightarrow$ $h) \times \mathcal{B}(h \rightarrow \gamma \gamma)]_{\mathrm{CMSSM}} /[\sigma(g g \rightarrow h) \times \mathcal{B}(h \rightarrow \gamma \gamma)]_{\mathrm{SM}} \leq 1.00$, and the $b \rightarrow s \gamma$ constraint has no impact. On the other hand, in panel (d), for $\mu<0$ and $\tan \beta=35$, we see that the $m_{\mathrm{h}}$ lower limit would allow values of $[\sigma(g g \rightarrow h) \times \mathcal{B}(h \rightarrow \gamma \gamma)]_{\mathrm{CMSSM}} /[\sigma(g g \rightarrow h) \times \mathcal{B}(h \rightarrow$ $\gamma \gamma)]_{\mathrm{SM}} \sim 0.50$, but this is strengthened to $\sim 0.80$ by the $b \rightarrow s \gamma$ constraint. In this case, there is always some suppression of the signal, and we find $[\sigma(g g \rightarrow h) \times \mathcal{B}(h \rightarrow$ $\gamma \gamma)]_{\mathrm{CMSSM}} /[\sigma(g g \rightarrow h) \times \mathcal{B}(h \rightarrow \gamma \gamma)]_{\mathrm{SM}} \leq 0.90$ if we impose $m_{\mathrm{H}}<117 \mathrm{GeV}$

This first survey shows (i) that the feared cancellations or other sources of suppression in $[\sigma(g g \rightarrow h) \times \mathcal{B}(h \rightarrow \gamma \gamma)]_{\mathrm{CMSSM}} /[\sigma(g g \rightarrow h) \times \mathcal{B}(h \rightarrow \gamma \gamma)]_{\mathrm{SM}}$ are relatively rare in the CMSSM, but (ii) they may occur for $\mu<0$ and large $\tan \beta$, in which case (iii) they may be avoided by imposing the cosmological and experimental constraints, notably (iv) $g_{\mu}-2$ and (v) $b \rightarrow s \gamma$.

We now explore the implications of varying the default parameters, starting in Fig. 2 with $m_{\mathrm{t}}$. We display the cases $\mu>0, \tan \beta=50$ and $m_{\mathrm{t}}=170 \mathrm{GeV}$ in panel (a) and $m_{\mathrm{t}}=180 \mathrm{GeV}$ in panel (b) $\mathrm{p}$. We see in panel (a) that $b \rightarrow s \gamma$ imposes $[\sigma(g g \rightarrow h) \times \mathcal{B}(h \rightarrow$ $\gamma \gamma)]_{\mathrm{CMSSM}} /[\sigma(g g \rightarrow h) \times \mathcal{B}(h \rightarrow \gamma \gamma)]_{\mathrm{SM}}>0.90$, whereas the $m_{\mathrm{h}}$ constraint alone would have allowed this ratio to fall to 0.85 . We see in panel (b) that this ratio could in principle be enhanced if $m_{\mathrm{t}}=180 \mathrm{GeV}$, although this possibility is disallowed by $b \rightarrow s \gamma$.

In the cases $\mu<0, \tan \beta=35$ and (c) $m_{\mathrm{t}}=170 \mathrm{GeV}$ and (d) $m_{\mathrm{t}}=180 \mathrm{GeV}$, we see again that values of $[\sigma(g g \rightarrow h) \times \mathcal{B}(h \rightarrow \gamma \gamma)]_{\mathrm{CMSSM}} /[\sigma(g g \rightarrow h) \times \mathcal{B}(h \rightarrow \gamma \gamma)]_{\mathrm{SM}}$ as low as 0.50 would be permitted by the $m_{\mathrm{h}}$ constraint, whereas the $b \rightarrow s \gamma$ constraint strengthens this lower limit to 0.80 in panel (c) and 0.85 in panel (d) of Fig. 2.

We now explore in Fig. 3 the implications of varying another default parameter, $A_{0}$,

\footnotetext{
${ }^{4}$ The irregularities in the cosmological region in panel (a) etc., and the separations between the dots in panel (b) etc. are due to the finite grid size used in our sampling of parameter space.

${ }^{5}$ The effects of varying $m_{\mathrm{t}}$ for $\tan \beta=10$ are less important, and are not discussed here.
} 


$$
[\sigma(g g \rightarrow h) \times \mathcal{B}(h \rightarrow \gamma \gamma)]_{\mathrm{CMSSM}} /[\sigma(g g \rightarrow h) \times \mathcal{B}(h \rightarrow \gamma \gamma)]_{\mathrm{SM}}
$$

(a)

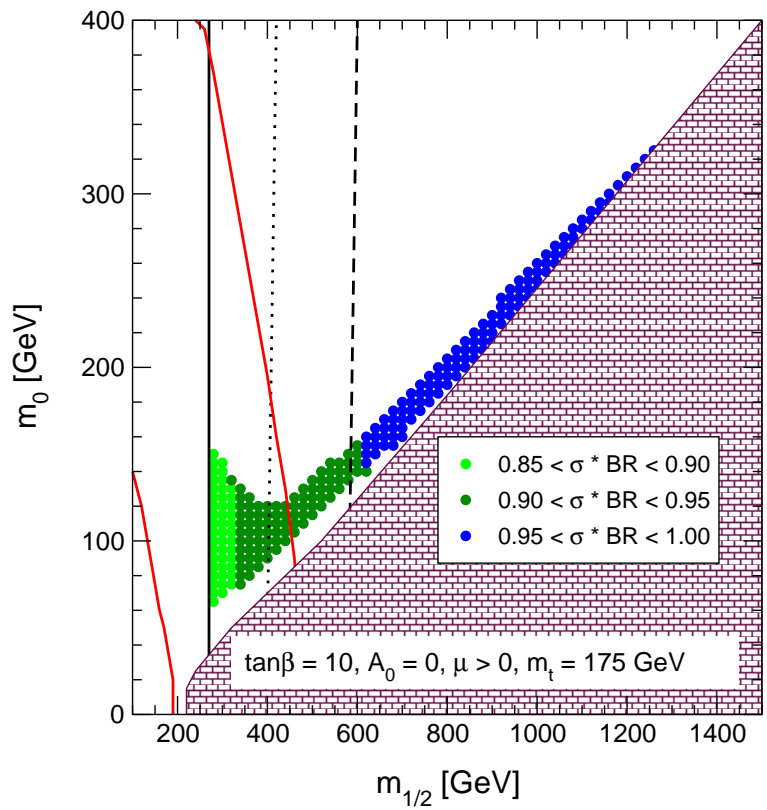

(c)

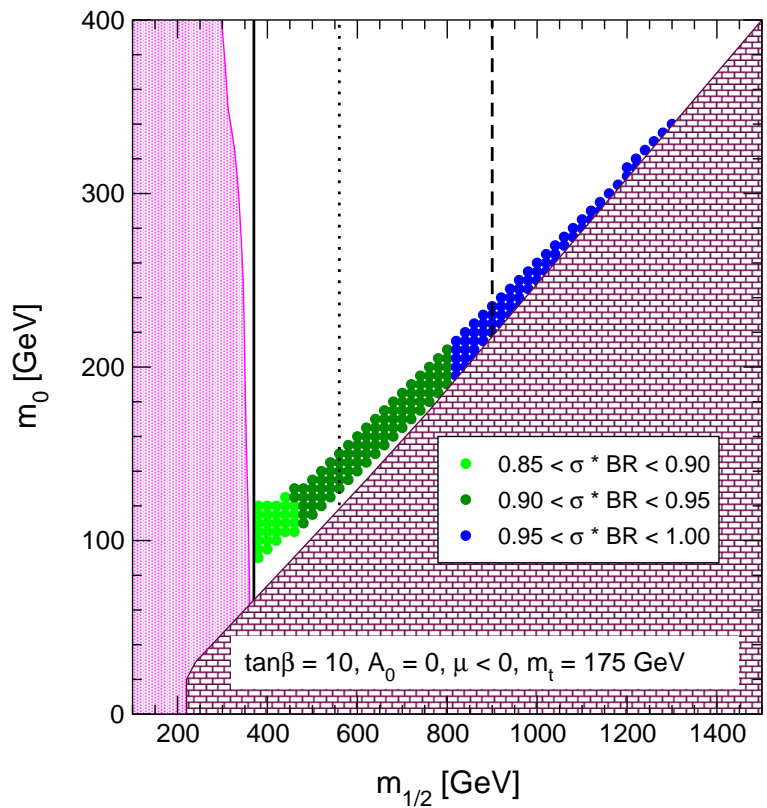

(b)

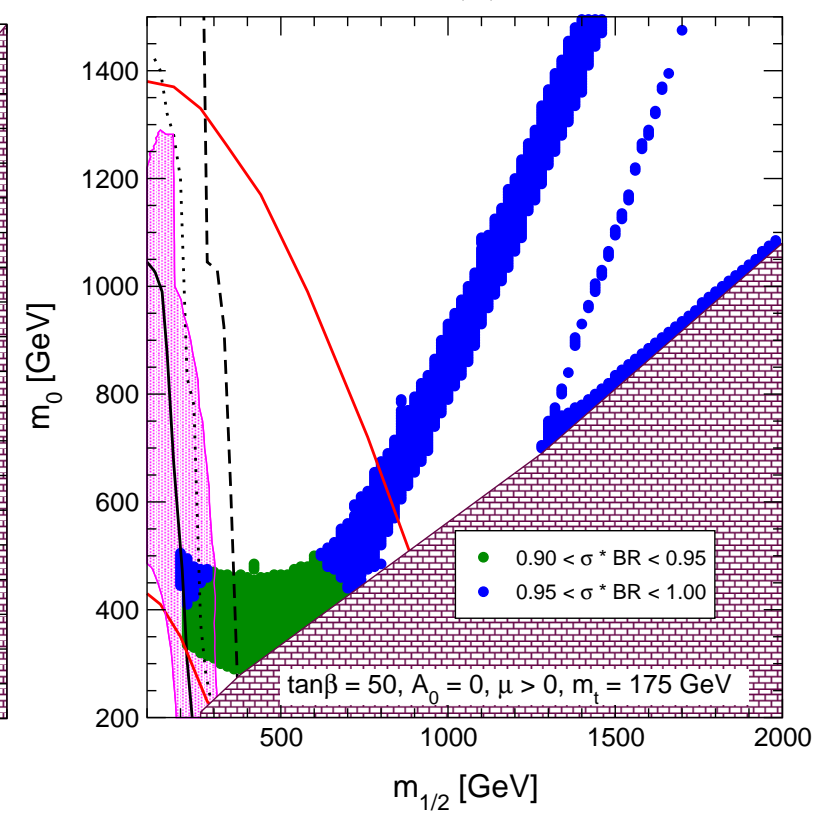

(d)

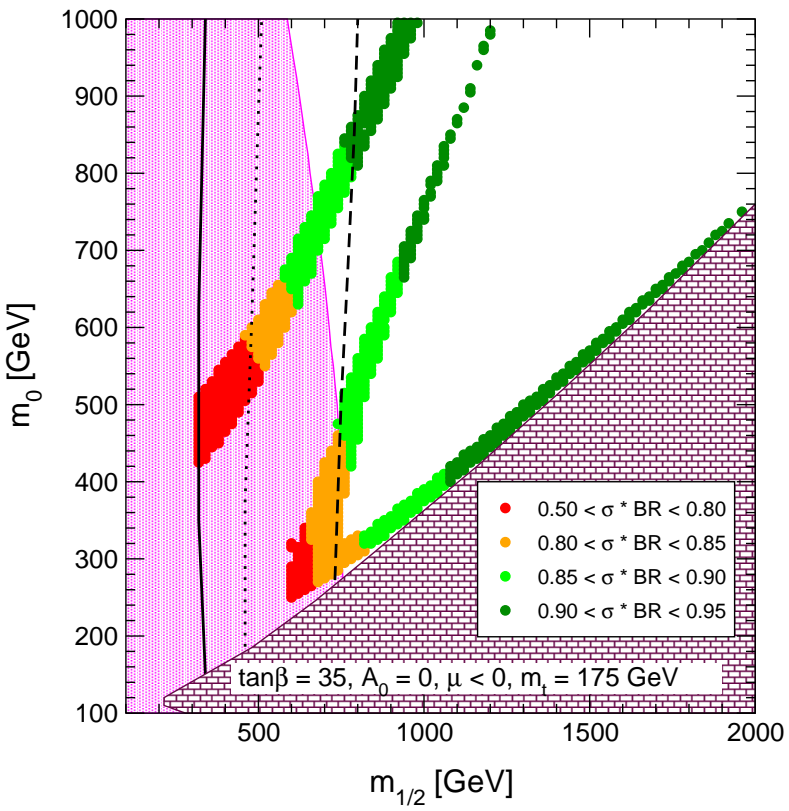

Figure 1: The cross section for production of the lightest $\mathcal{C} \mathcal{P}$-even $C M S S M$ Higgs boson in gluon fusion and its decay into a photon pair, $\sigma(g g \rightarrow h) \times \mathcal{B}(h \rightarrow \gamma \gamma)$, normalized to the SM value with the same Higgs mass, is given in the $\left(m_{1 / 2}, m_{0}\right)$ planes for $\mu>0, \tan \beta=10,50$ (upper row) and for $\mu<0, \tan \beta=10,35$ (lower row). In all plots $A_{0}=0$ and $m_{\mathrm{t}}=175$ GeV has been used. The diagonal (red) solid lines in panels ( $a$ ) and (b) are the $\pm 2-\sigma$ contours for $g_{\mu}-2$ : the whole parameter space in the $\mu<0$ plots is excluded by the $g_{\mu}-2$ constraint 11, 19]. The near-vertical solid, dotted and dashed (black) lines are the $m_{h}=113,115,117$ GeV contours. The light shaded (pink) regions are excluded by $b \rightarrow s \gamma$ 17,8]. The (brown) bricked regions are excluded since in these regions the $L S P$ is the charged $\tilde{\tau}_{1}$. 


$$
[\sigma(g g \rightarrow h) \times \mathcal{B}(h \rightarrow \gamma \gamma)]_{\mathrm{CMSSM}} /[\sigma(g g \rightarrow h) \times \mathcal{B}(h \rightarrow \gamma \gamma)]_{\mathrm{SM}}
$$

(a)

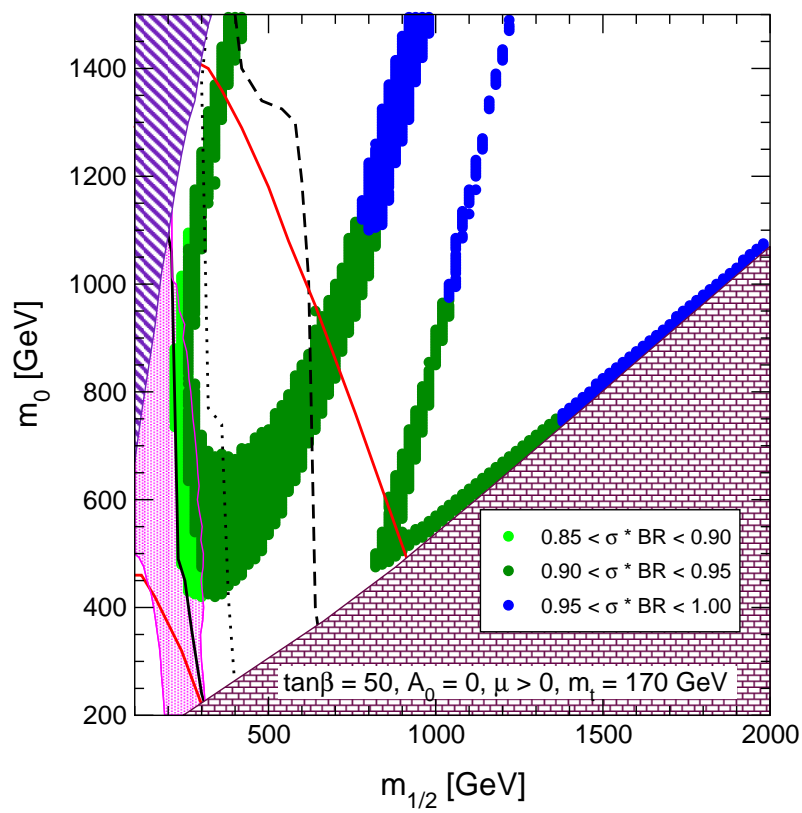

(c)

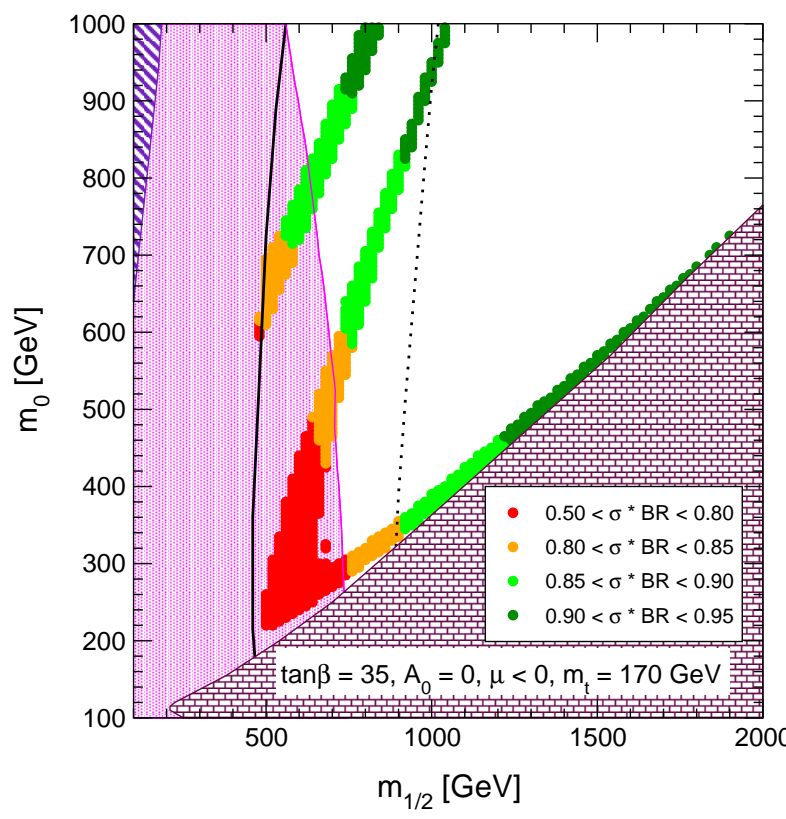

(b)

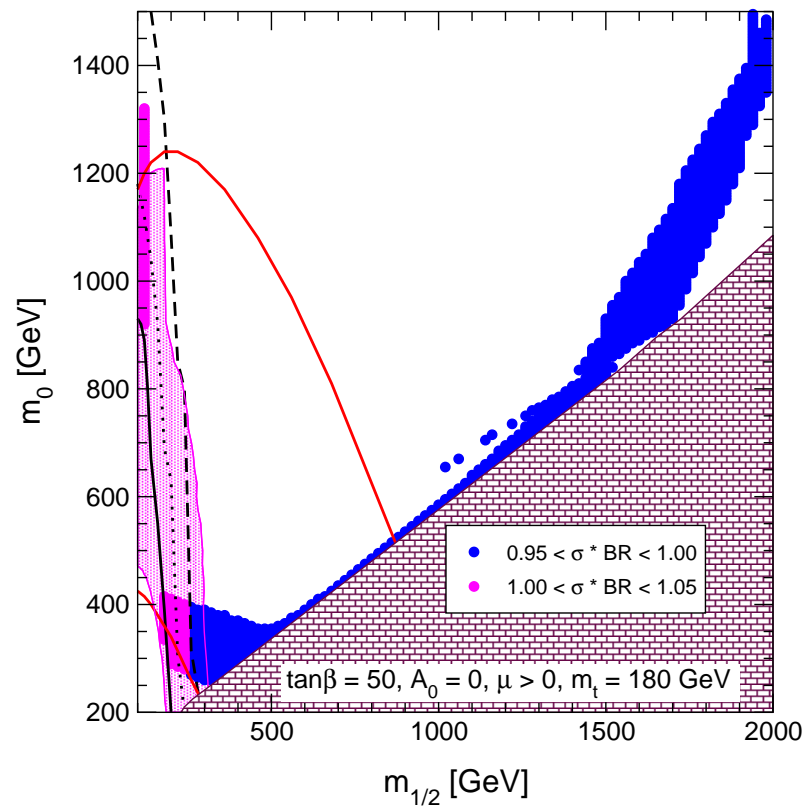

(d)

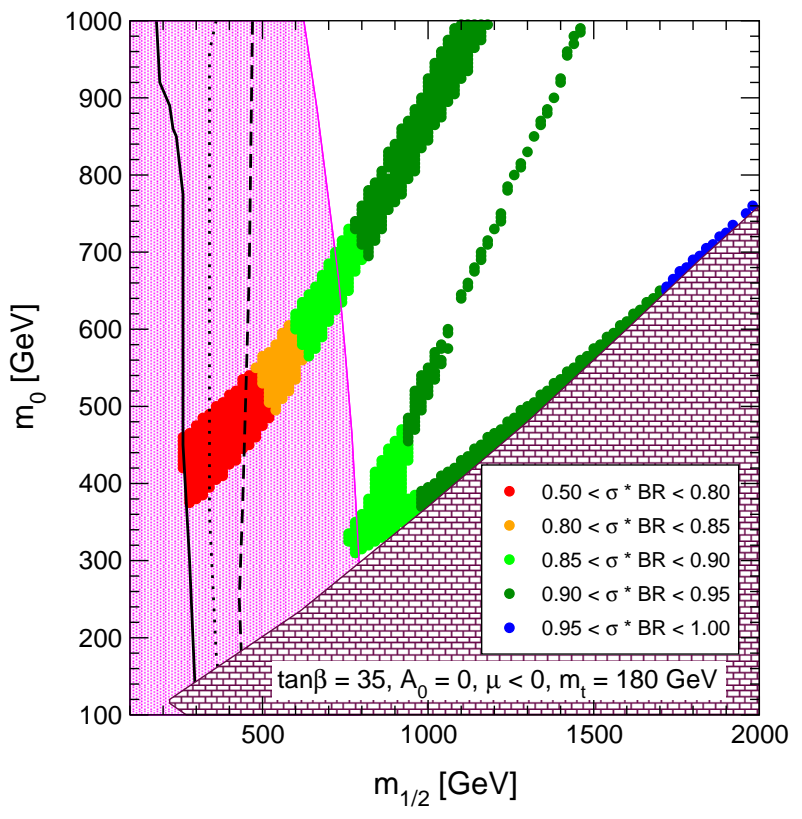

Figure 2: The cross section for production of the lightest $\mathcal{C} \mathcal{P}$-even $M S S M$ Higgs boson in gluon fusion and its decay into a photon pair, $\sigma(g g \rightarrow h) \times \mathcal{B}(h \rightarrow \gamma \gamma)$, normalized to the SM value with the same Higgs mass, is given in the $\left(m_{1 / 2}, m_{0}\right)$ planes for $\mu>0, \tan \beta=50$ and $m_{\mathrm{t}}=170,180 \mathrm{GeV}$ (upper row) as well as for $\mu<0, \tan \beta=35$ and $m_{\mathrm{t}}=170,180 \mathrm{GeV}$ (lower row). In all plots $A_{0}=0$ has been used, and the notation is the same as in Fig. 1 . The striped regions at small values of $m_{1 / 2} / m_{0}$ in panels (a) and (c) are excluded by the constraint of radiative electroweak symmetry breaking. 
considering first the cases $\mu>0, \tan \beta=50$ and (a) $A_{0}=-2 \times m_{1 / 2}$ and (b) $A_{0}=+m_{1 / 2}$ व We have also considered the case $A_{0}=+2 \times m_{1 / 2}$, but did not find any significant allowed region surviving the constraints from cosmology and $b \rightarrow s \gamma$. We see in panel (a) that the $m_{\mathrm{h}}$ and $b \rightarrow s \gamma$ constraints are essentially equivalent for this sign of $A_{0}$, and each impose $[\sigma(g g \rightarrow h) \times \mathcal{B}(h \rightarrow \gamma \gamma)]_{\mathrm{CMSSM}} /[\sigma(g g \rightarrow h) \times \mathcal{B}(h \rightarrow \gamma \gamma)]_{\mathrm{SM}}>0.90$. In the case of $A_{0}=+m_{1 / 2}$, shown in panel (b), there are again no cancellations.

The cases $\mu<0, \tan \beta=35$ and (c) $A_{0}=-2 \times m_{1 / 2}$ and (d) $A_{0}=+m_{1 / 2}$ exhibit more variation. In the former case, $[\sigma(g g \rightarrow h) \times \mathcal{B}(h \rightarrow \gamma \gamma)]_{\mathrm{CMSSM}} /[\sigma(g g \rightarrow h) \times \mathcal{B}(h \rightarrow$ $\gamma \gamma)]_{\mathrm{SM}}<0.80$ would be allowed by $m_{\mathrm{h}}$ but not by $b \rightarrow s \gamma$. On the other hand, in panel (d) for $A_{0}=+m_{1 / 2}$, we see that values of this ratio even below 0.50 are allowed a priori. However, this rises to 0.80 once we impose the $b \rightarrow s \gamma$ constraint, and we recall that this and all $\mu<0$ cases are excluded by the $g_{\mu}-2$ measurement.

Apart from this possibility for reducing the $h \rightarrow \gamma \gamma$ signal, which involves discarding some of the principal experimental constraints on the CMSSM, we conclude that the $h \rightarrow \gamma \gamma$ mode should be (almost) as easy to detect at the LHC as the corresponding signal for a SM Higgs boson of the same mass.

In view of their potential interest at the Tevatron as well as at the LHC, we have also considered the strengths of the signals for $W^{ \pm} / Z^{0}+h, h \rightarrow \bar{b} b$ and $\bar{t} t+h, h \rightarrow \bar{b} b$. We do not distinguish between the results for these two channels, as we find that, within the constraints we impose on the CMSSM, the $W^{ \pm} W^{\mp}+h, Z^{0} Z^{0}+h$ and $\bar{t} t+h$ couplings differ insignificantly from those in the SM. Thus the differences in the signals from those in the $\mathrm{SM}$ are essentially controlled by the differences in $\mathcal{B}(h \rightarrow \bar{b} b)$. It is well known that this is generically enhanced in the MSSM relative to the SM, particularly at large $\tan \beta$, and this is reflected in our results.

In Fig. 4 the results for the two channels are shown for the default case $A_{0}=0$ and $m_{\mathrm{t}}=175 \mathrm{GeV}$. Panels (a) and (b) are for $\mu>0$ and $\tan \beta=10,50$, respectively, while panels (c) and (d) show the case $\mu<0$ and $\tan \beta=10,35$, respectively. As expected, for all parameter values in Fig. $⿴$ ⿶ we find a slight enhancement of up to $5 \%$ in the $W^{ \pm} / Z^{0}+h, h \rightarrow \bar{b} b$ and $\bar{t} t+h, h \rightarrow \bar{b} b$ channels compared to the SM case. An enhancement by up to $10 \%$ occurs for $\mu<0$ and $\tan \beta=35$, but the corresponding parameter region is disfavoured by the $b \rightarrow s \gamma$ constraint, not to mention $g_{\mu}-2$. We do not display results for $A_{0} \neq 0$ and $m_{\mathrm{t}}=170,180 \mathrm{GeV}$ for these channels, since for all parameter regions allowed by the $b \rightarrow s \gamma$ constraint we find the same results as in Fig. 4 , i.e. an enhancement compared to the SM value of up to $5 \%$.

\footnotetext{
${ }^{6}$ The effects of varying $A_{0}$ for $\tan \beta=10$ are also less important.
} 


$$
[\sigma(g g \rightarrow h) \times \mathcal{B}(h \rightarrow \gamma \gamma)]_{\mathrm{CMSSM}} /[\sigma(g g \rightarrow h) \times \mathcal{B}(h \rightarrow \gamma \gamma)]_{\mathrm{SM}}
$$

(a)

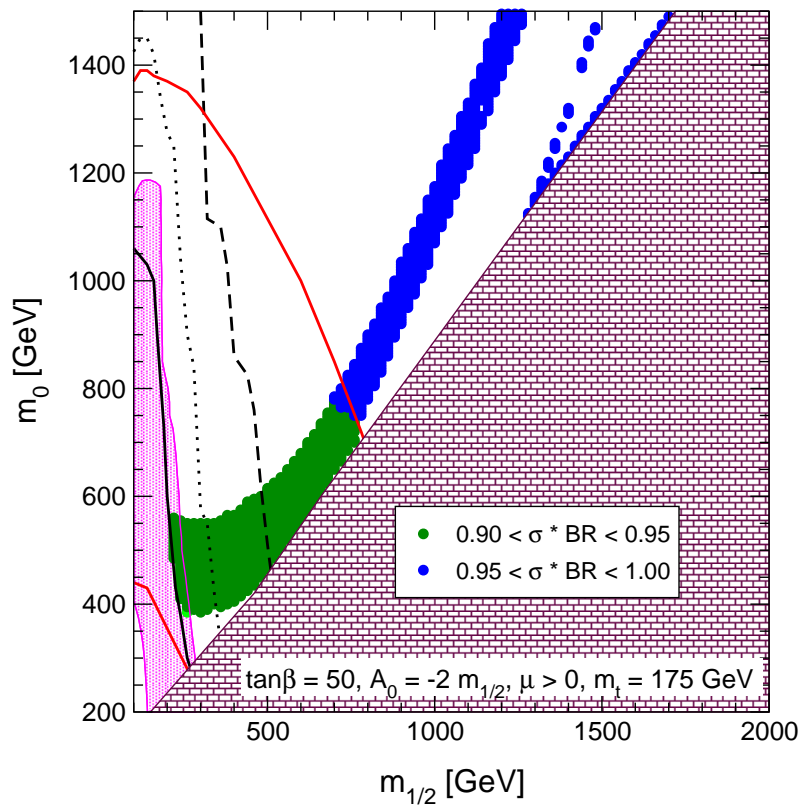

(c)

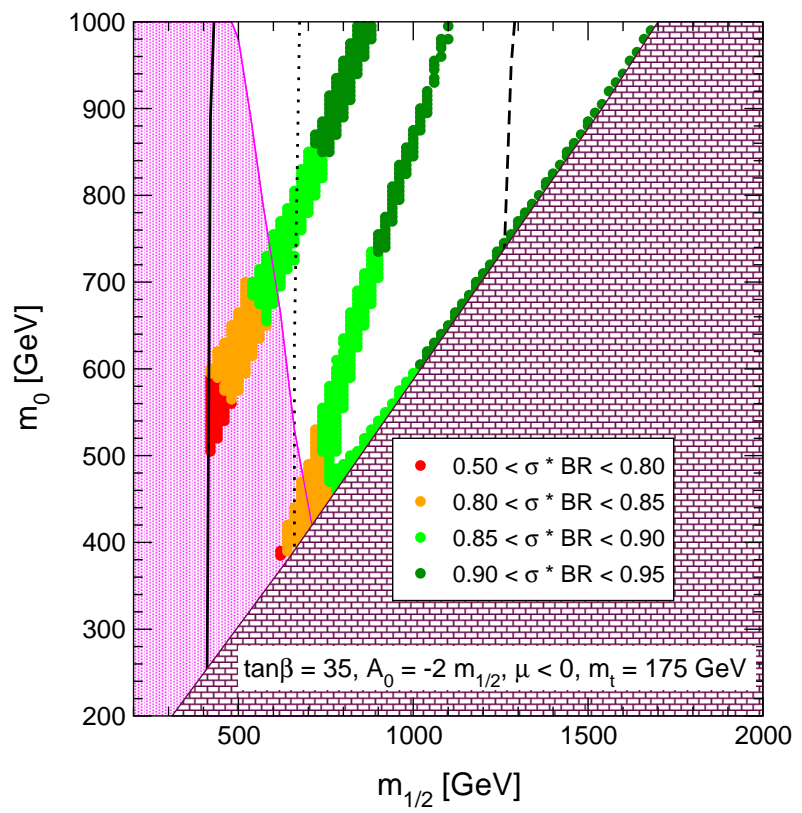

(b)

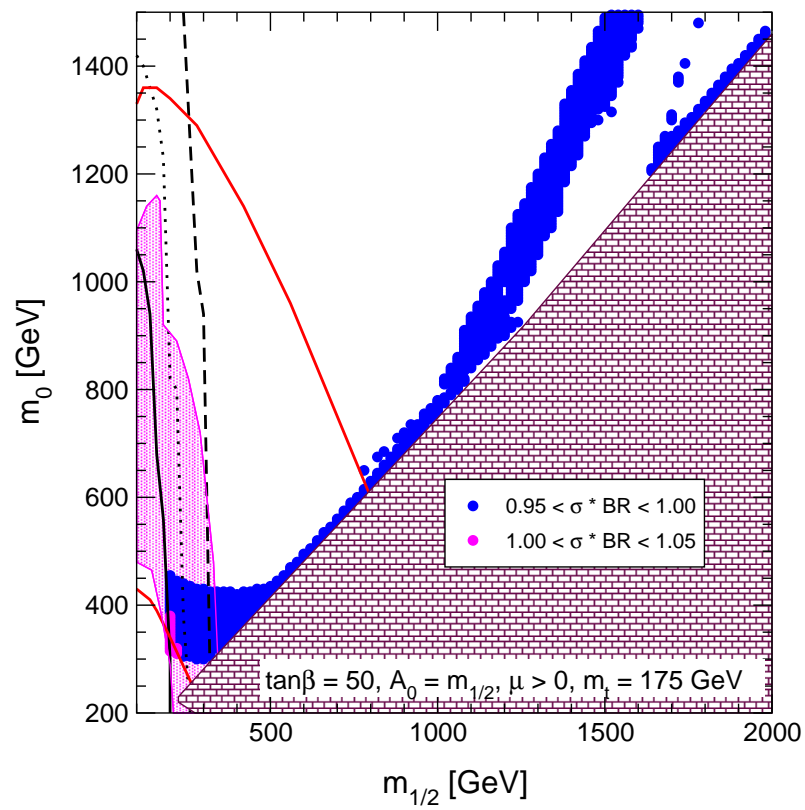

(d)

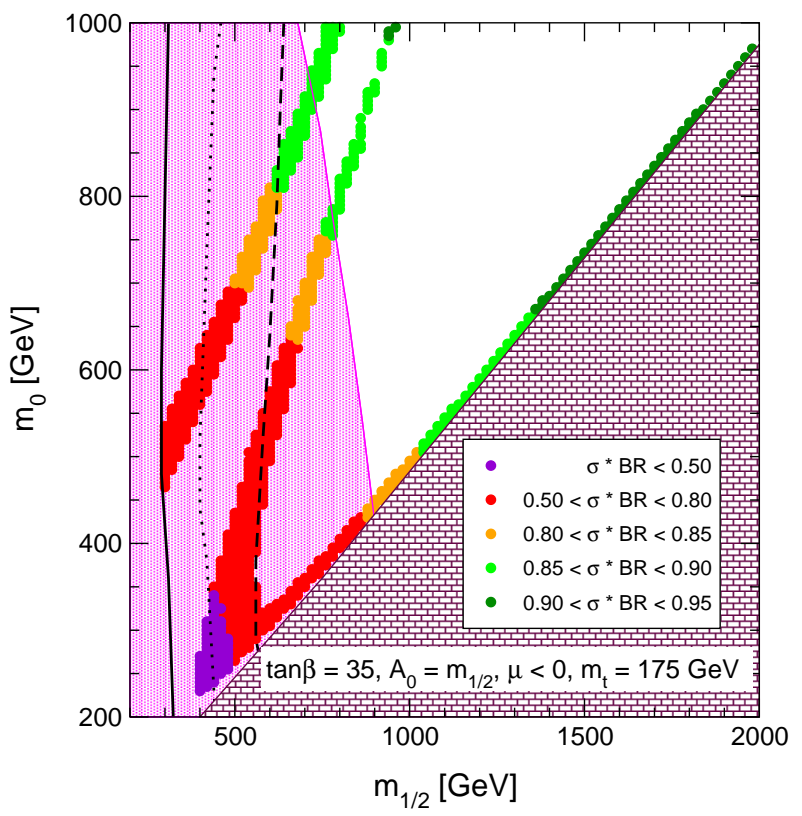

Figure 3: The cross section for production of the lightest $\mathcal{C} \mathcal{P}$-even $M S S M$ Higgs boson in gluon fusion and its decay into a photon pair, $\sigma(g g \rightarrow h) \times \mathcal{B}(h \rightarrow \gamma \gamma)$, normalized to the SM value with the same Higgs mass, is given in the $\left(m_{1 / 2}, m_{0}\right)$ planes for $\mu>0, \tan \beta=50$ and $A_{0}=-2 m_{1 / 2},+m_{1 / 2}$ (upper row) as well as for $\mu<0, \tan \beta=35$ and $A_{0}=-2 m_{1 / 2},+m_{1 / 2}$ (lower row). In all plots $m_{\mathrm{t}}=175 \mathrm{GeV}$ has been used, and the notation is the same as in Fig. 团. 


$$
\left[\sigma\left(W^{ \pm}, Z^{0} / \bar{t} t+h\right) \times \mathcal{B}(h \rightarrow \bar{b} b)\right]_{\mathrm{CMSSM}} /\left[\sigma\left(W^{ \pm}, Z^{0} / \bar{t} t+h\right) \times \mathcal{B}(h \rightarrow \bar{b} b)\right]_{\mathrm{SM}}
$$

(a)

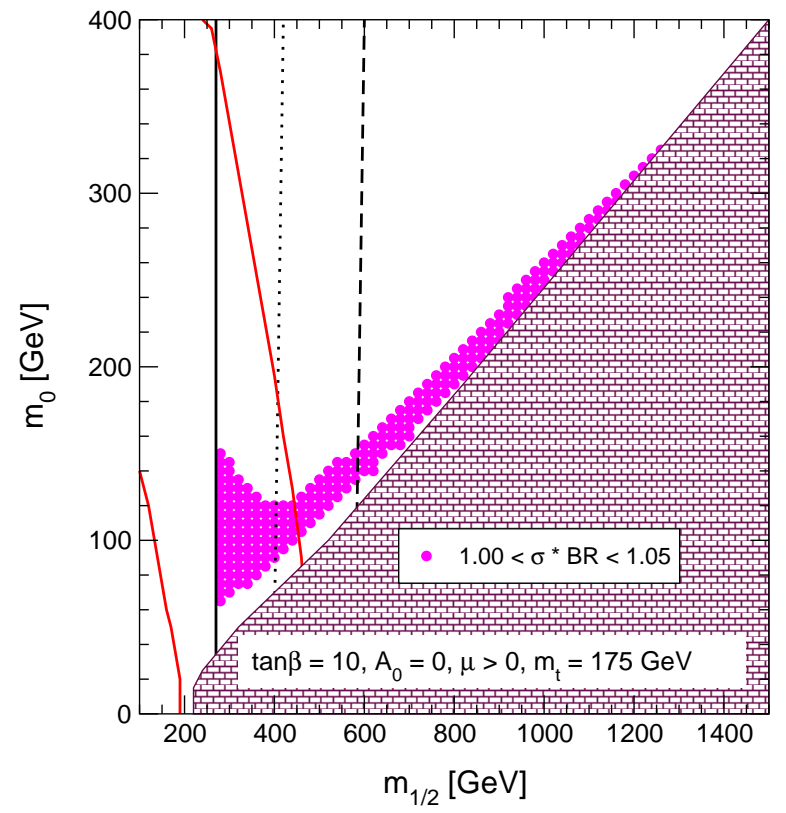

(c)

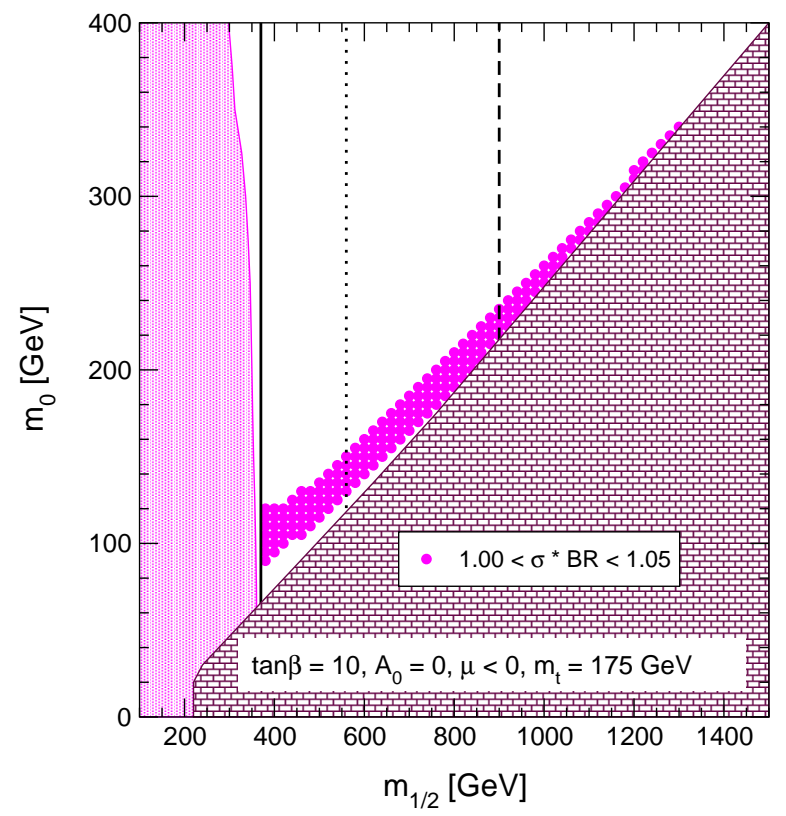

(b)

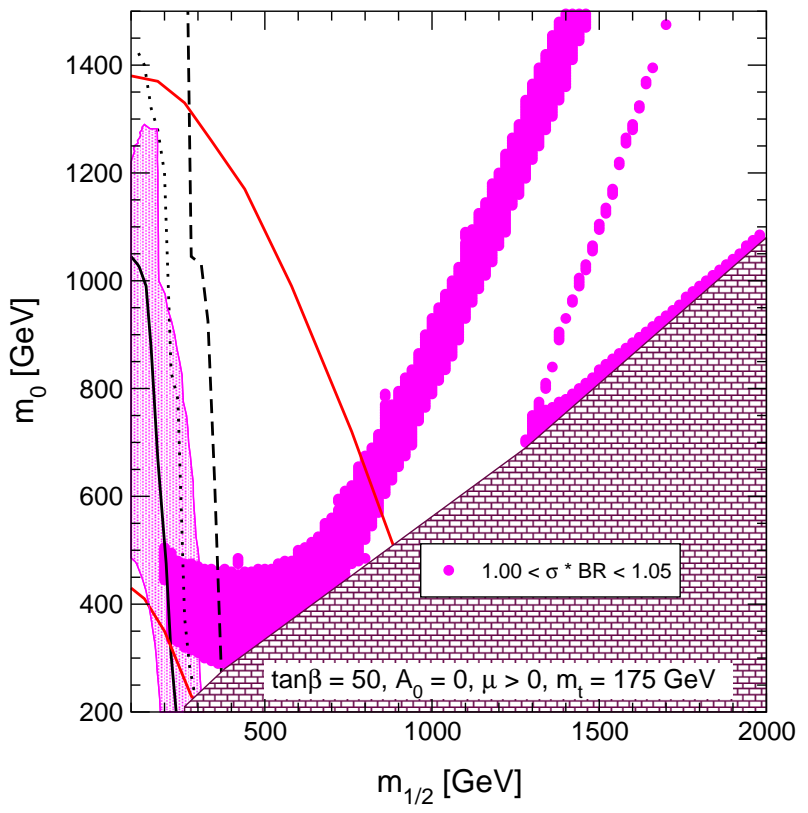

(d)

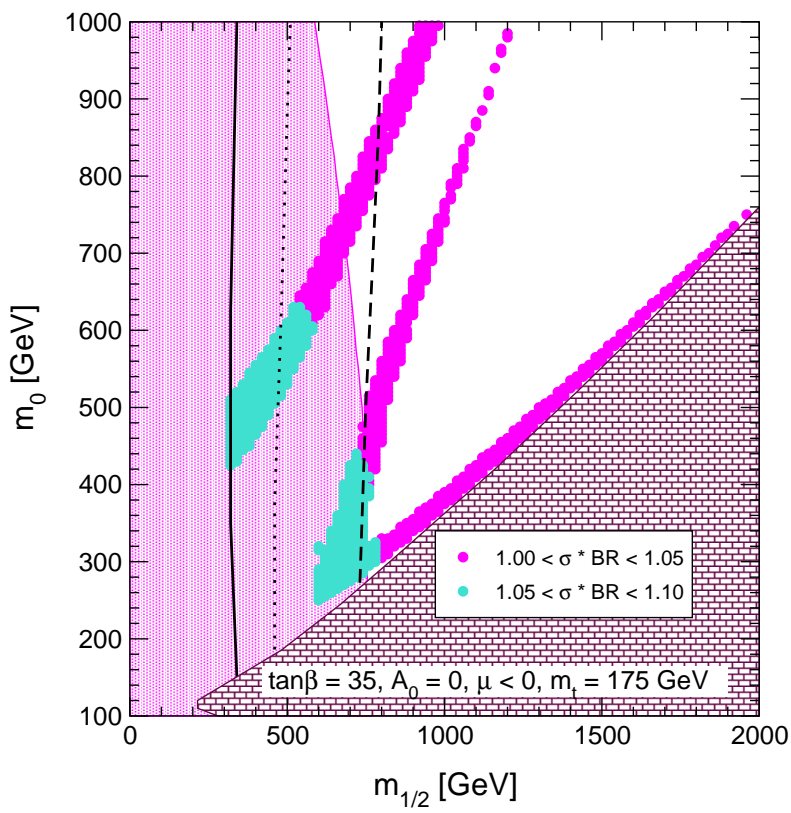

Figure 4: The cross section for production of the lightest $\mathcal{C P}$-even MSSM Higgs boson in association with a $\bar{t} t$ pair or with $W^{ \pm} / Z$, followed by decay into $\bar{b} b$, are given normalized to the SM value with the same Higgs mass. The results are displayed in the $\left(m_{1 / 2}, m_{0}\right)$ planes for $\mu>0, \tan \beta=10,50$ (upper row) and for $\mu<0, \tan \beta=10,35$ (lower row). In all plots $A_{0}=0$ and $m_{\mathrm{t}}=175$ GeV has been used, and the notation is the same as in Fig. 囵. 
Could one, in principle, distinguish a CMSSM Higgs boson from a SM Higgs boson of the same mass, simply by measuring its production cross section? The present LHC goal for measuring luminosity at the parton-parton level is $\pm 5 \%$, and the statistical precision in the $h \rightarrow \gamma \gamma$ channel might approach 1\%. Thus, if the theoretical error could be neglected, there could be a 2- $\sigma$ experimental difference between the strengths of the CMSSM and SM signals, which might be strengthened if the luminosity precision goal could be bettered. In the case of the $W^{ \pm} / Z^{0}+h, h \rightarrow \bar{b} b$ and $\bar{t} t+h, h \rightarrow \bar{b} b$ channels, there is a further experimental error of about $5 \%$ associated with the background subtractions. Thus, distinguishing between the strengths expected in the CMSSM and the SM does not appear feasible in these channels.

We conclude that the lightest CMSSM Higgs boson $h$ should be almost as easy to see as the Standard Model Higgs boson, if one accepts all the present experimental and cosmological constraints. In particular, the previous analyses of the prospects for Higgs searches at the LHC and Tevatron indicate that the $h$ boson should be discoverable with about $10 \mathrm{fb}^{-1}$ of luminosity at the LHC [3]. If its mass is about $115 \mathrm{GeV}$, i.e. close to the current SM exclusion bound, it is likely also to be discoverable with $15 \mathrm{fb}^{-1}$ of luminosity at the Tevatron collider [2].

\section{Acknowledgments}

We thank Geri Ganis for useful information and Fabiola Gianotti for an interesting discussion. The work of K.A.O. was partially supported by DOE grant DE-FG02-94ER-40823.

\section{References}

[1] ALEPH collaboration, R. Barate et al., Phys. Lett. B495 (2000) 1;

L3 collaboration, M. Acciarri et al., Phys. Lett. B495 (2000) 18;

DELPHI collaboration, P. Abreu et al., Phys. Lett. B 499 (2001) 23;

OPAL collaboration, G. Abbiendi et al., Phys. Lett. B499 38.

For a preliminary compilation of the LEP data presented on Nov. 3rd, 2000, see:

P. Igo-Kemenes, for the LEP Higgs working group, http://lephiggs.web.cern.ch/LEPHIGGS/talks/index.html.

For a recent compilation of other LEP search data, as presented on Sept. 5th, 2000, see: T. Junk, hep-ex/0101015.

[2] M. Carena et al., Report of the Tevatron Higgs working group, hep-ph/0010338; P. Sinervo, Talk at the Conference on Higgs and Supersymmetry, Orsay, March 19-22, 
2001

http://ww2.lal.in2p3.fr/actualite/conferences/higgs2001/slides/index.htm.

[3] ATLAS Collaboration, Detector and Physics Performance Technical Design Report, http://atlasinfo.cern.ch/Atlas/GROUPS/PHYSICS/TDR/access.html;

CMS Collaboration, http://cmsinfo.cern.ch/Welcome.html/CMSdocuments/CMSplots/;

F. Gianotti, Talk at the Conference on Higgs and Supersymmetry, Orsay, March 19-22, 2001 ,

http://ww2.lal.in2p3.fr/actualite/conferences/higgs2001/slides/index.htm.

[4] Y. Okada, M. Yamaguchi and T. Yanagida, Prog. Theor. Phys. 85 (1991) 1; J. Ellis, G. Ridolfi and F. Zwirner, Phys. Lett. B 257 (1991) 83; H. E. Haber and R. Hempfling, Phys. Rev. Lett. 66 (1991) 1815; M. Carena, J. R. Espinosa, M. Quiros and C. E. Wagner, Phys. Lett. B 355 (1995) 209; M. Carena, M. Quiros and C. E. Wagner, Nucl. Phys. B 461 (1996) 407; H.E. Haber, R. Hempfling and A.H. Hoang, Zeit. für Phys. C75 (1997) 539; S. Heinemeyer, W. Hollik and G. Weiglein, Eur. Phys. Jour. C9 (1999) 343; J. R. Espinosa and R. Zhang, Nucl. Phys. B 586, 3 (2000) hep-ph/0003246.

[5] E. Richter-Wạs, D. Froidevaux, F. Gianotti, L. Poggioli, D. Cavalli and S. Resconi, Int. J. Mod. Phys. A13 (1998) 1371.

[6] M. Carena, S. Mrenna and C. E. Wagner, Phys. Rev. D60 (1999) 075010 and Phys. Rev. D 62 (2000) 055008.

[7] CLEO Collaboration, M.S. Alam et al., Phys. Rev. Lett. 74 (1995) 2885 as updated in S. Ahmed et al., CLEO CONF 99-10; K. Abe et al., Belle Collaboration, hepex/0103042.

[8] G. Degrassi, P. Gambino and G. F. Giudice, JHEP 0012 (2000) 009; see also M. Carena, D. Garcia, U. Nierste and C. E. Wagner, hep-ph/0010003.

[9] J. Ellis, J.S. Hagelin, D.V. Nanopoulos, K.A. Olive and M. Srednicki, Nucl. Phys. B238 (1984) 453; see also H. Goldberg, Phys. Rev. Lett. 50 (1983) 1419.

[10] J. Ellis, T. Falk, G. Ganis, K. A. Olive and M. Srednicki, hep-ph/0102098.

[11] H. N. Brown et al., Muon $g_{\mu}-2$ Collaboration, Phys. Rev. Lett. 86 (2001) 2227. 
[12] J. Ellis, D. V. Nanopoulos and K. A. Olive, hep-ph/0102331; for other recent papers on the CMSSM interpretation of $g_{\mu}-2$, see L. Everett, G. L. Kane, S. Rigolin and L. Wang, hep-ph/0102145; J. L. Feng and K. T. Matchev, hep-ph/0102146; E. A. Baltz and P. Gondolo, hep-ph/0102147; U. Chattopadhyay and P. Nath, hep-ph/0102157; S. Komine, T. Moroi and M. Yamaguchi, hep-ph/0102204; J. Hisano and K. Tobe, hepph/0102315. R. Arnowitt, B. Dutta, B. Hu and Y. Santoso, hep-ph/0102344; K. Choi, K. Hwang, S. K. Kang, K. Y. Lee and W. Y. Song, hep-ph/0103048; S. P. Martin and J. D. Wells, hep-ph/0103067; S. Komine, T. Moroi and M. Yamaguchi, hep-ph/0103182; H. Baer, C. Balazs, J. Ferrandis and X. Tata, hep-ph/0103280.

[13] H. M. Georgi, S. L. Glashow, M. E. Machacek and D. V. Nanopoulos, Phys. Rev. Lett. 40 (1978) 692; S. Dawson, Nucl. Phys. B359 (1991) 283; A. Djouadi, M. Spira and P. Zerwas, Phys. Lett. B264 (1991) 440; D. Graudenz, M. Spira and P. Zerwas, Phys. Rev. Lett. 70 (1993) 1372; M. Spira, A. Djouadi, D. Graudenz and P. Zerwas, Nucl. Phys. B453 (1995) 17; A. Djouadi, Phys. Lett. B 435, 101 (1998) hepph/9806315]; G. Belanger, F. Boudjema and K. Sridhar, Nucl. Phys. B 568, 3 (2000) hep-ph/9904348]; G. Belanger, F. Boudjema, F. Donato, R. Godbole and S. RosierLees, Nucl. Phys. B 581, 3 (2000) hep-ph/0002039; S. Catani, D. de Florian and M Grazzini, hep-ph/0102227; R. Harlander and W. Kilgore, hep-ph/0102241.

[14] J. Ellis, M. K. Gaillard and D. V. Nanopoulos, Nucl. Phys. B106 (1976) 292.

[15] S. L. Glashow, D. V. Nanopoulos and A. Yildiz, Phys. Rev. D18 (1978) 1724.

[16] S. Heinemeyer, W. Hollik and G. Weiglein, Comput. Phys. Commun. 124 (2000) 76; hep-ph/0002213; the FeynHiggs code is available from http://www.feynhiggs.de.

[17] S. Heinemeyer, W. Hollik and G. Weiglein, Phys. Rev. D58 (1998) 091701; Phys. Lett. B440 (1998) 296; Eur. Phys. Jour. C16 (2000) 139.

[18] A. Djouadi, J. Kalinowski and M. Spira, Comput. Phys. Commun. 108 (1998) 56.

[19] S. P. Martin and M. T. Vaughn, Phys. Rev. D50, 2282 (1994).

[20] S. Heinemeyer, W. Hollik and G. Weiglein, hep-ph/9910283; J. R. Espinosa and R. Zhang, JHEP 0003, 026 (2000) hep-ph/9912236; M. Carena, H. Haber, S. Heinemeyer, W. Hollik, C. Wagner and G. Weiglein, Nucl. Phys. B580 (2000) 29.

[21] The LEP working group for Higgs boson searches, LHWG Note 2001-2, http://lephiggs .web.cern.ch/LEPHIGGS. 